\title{
Mesoporous polypyrrole-based graphene nanosheets anchoring redox polyoxometalate for all-solid-state micro-supercapacitors with enhanced volumetric capacitance
}

\author{
Jieqiong Qin ${ }^{1,2}$, Feng Zhou ${ }^{1}$, Han Xiao ${ }^{1}$, Ruyi Ren ${ }^{1}$ and Zhong-Shuai $\mathrm{Wu}^{{ }^{1 *}}$
}

\begin{abstract}
Micro-supercapacitors (MSCs) have emerged as one competitive candidate of high-performance, flexible, safe, portable and wearable energy storage devices. However, improving their electrochemical performance from electrode materials to assembled devices still remains huge challenges. Here, we for the first time synthesized two-dimensional (2D), ultrathin, mesoporous polypyrrole-based graphene nanosheets uniformly anchored with redox polyoxometalate (mPPy@rGO-POM) by soft template approach. Further, using a layer-by-layer deposition and mask-assisted technique, the compactly stacked and sandwich-like hybrid film (mPGM) based on pseudocapacitive mPPy@rGO-POM nanosheets and electrochemically exfoliated graphene was directly fabricated as binder- and additive-free interdigital electrodes for all-solid-state planar micro-supercapacitors (mPGM-MSCs). Notably, the resulted mPGM-MSCs exhibited outstanding areal capacitance $\left(115 \mathrm{mF} \mathrm{cm}^{-2}\right)$, remarkably enhanced volumetric capacitance $\left(137 \mathrm{~F} \mathrm{~cm}^{-3}\right.$ at $\left.1 \mathrm{mV} \mathrm{s}^{-1}\right)$ in comparison with MSCs based on the films of mPPy@rGO without POM anchoring (95 $\left.\mathrm{F} \mathrm{cm}^{-3}\right)$, and non-porous polypyrrole-graphene $\left(68 \mathrm{~F} \mathrm{~cm}^{-3}\right)$. Further, mPGM-MSCs disclosed robust mechanical flexibility with $\sim 96 \%$ of capacitance retention at a highly bending angle of $180^{\circ}$, and impressive parallel or serial interconnection for boosting capacitance or voltage output. As a consequence, our proposed strategy of filling the redox species into mesoporous graphene and other 2D nanosheets will open up new ways to manufacture high-compact and flexible energy storage devices ranging from supercapacitors to batteries.
\end{abstract}

Keywords: mesoporous, graphene, redox, all-solid-state, microsupercapacitors

\section{INTRODUCTION}

The ever-increasing consumption of energy demands highly efficient energy storage devices, such as supercapacitors that provide high power density, long-term cycle life, and fast charge and discharge process [1-5]. Recently, micro-supercapacitors (MSCs) with multiple compatible characteristics of lightweight, tailored size, robust mechanical flexibility, and ultrahigh power delivery have attracted tremendous attentions [6-8]. Despite dramatic developments in innovating nanostructured electrode materials and fabricating novel devices, there are still enormous challenges in realizing MSCs with high volumetric capacitance and energy density $[9,10]$. Therefore, the exploitation of high-compact film electrode materials with developed electronic and ionic conducting networks is crucial for MSCs, but still underdeveloped in this research field [11-14].

Conducting polymers, such as polypyrrole (PPy) have been extensively utilized in energy storage devices (e.g., MSCs) [15-17]. Unfortunately, the incident volume change and unsatisfied electrical conductivity of conducting polymers result in low power density, poor rate capability and cyclability for MSCs. To overcome these drawbacks, the introduction of mesoporous structure and conductive network (e.g., graphene) to construct twodimensional (2D) graphene-based mesoporous polymer nanosheets with high specific surface area, ultra-thinness, interconnected pore structure, good electrical conductivity and excellent flexibility could remarkably improve their electrochemical performance for MSCs [18-

\footnotetext{
${ }^{1}$ Dalian National Laboratory for Clean Energy, Dalian Institute of Chemical Physics, Chinese Academy of Sciences, Dalian 116023, China

${ }^{2}$ University of Chinese Academy of Sciences, Beijing 100049, China

* Corresponding author (email: wuzs@dicp.ac.cn)
} 
20]. On the other hand, the presence of mesopores in such $2 \mathrm{D}$ nanosheets is favorable for the anchoring of highly redox species in order to construct densely compact films for MSCs with enhanced volumetric performance.

Polyoxometalates (POMs), as redox-active molecular metal oxide clusters containing high-valent transition metals (e.g., V, Mo and W), possess a variety of structures, sizes and compositions [21]. In particular, Keggin cluster $\left[\mathrm{PMo}_{12} \mathrm{O}_{40}\right](\mathrm{POM})$ has been reported as a prospective candidate for supercapacitors with high capacitance, due to its abundance, stable redox states, fast and reversible multi-electron transfer reactions [22,23]. However, POMs exhibit intrinsically poor conductivity and high solubility in aqueous and polar organic solvents. In general, POMs require anchoring onto a chemically stable, large surface area substrates ranging from conducting polymers to graphene, activated carbons or carbon nanotubes to form hybrid materials, in order to prevent the dissolution of POMs into electrolyte, provide the necessary electrical conductivity and utilize the high pseudocapacitance [24-26]. Nevertheless, rational design of anchoring POMs on 2D mesoporous graphene nanosheets for compact MSCs has not yet been reported.

Herein, we reported the designed synthesis of $2 \mathrm{D}$ mesoporous polypyrrole-based graphene (mPPy@rGO) nanosheets with open and wormlike mesopores, which were well suitable for the homogeneous and efficient anchoring of redox POM clusters. After POM anchoring, the asobtained 2D hybrid nanosheets (mPPy@rGO-POM) remained uniform, ultrathin and mesoporous structure and presented notable multiple redox peaks, indicative of strong pseudocapacitive behavior from POM clusters. Further, employing layer-by-layer deposition and interdigital mask-assisted techniques, we directly constructed sandwich-like staked-layer hybrid film (mPGM) with pseudocapacitive mPPy@rGO-POM and capacitive electrochemically exfoliated graphene (EG) nanosheets for all-solid-state MSCs (mPGM-MSCs). Remarkably, the resulted all-solid-state mPGM-MSCs with a polymer gel electrolyte exhibited superior areal capacitance (115 $\mathrm{mF} \mathrm{cm}{ }^{-2}$ ), enhanced volumetric capacitance $\left(137 \mathrm{~F} \mathrm{~cm}^{-3}\right)$, unprecedented flexibility with $\sim 96 \%$ of initial capacitance even bended at $180^{\circ}$, and efficient integration through parallel and serial interconnection.

\section{EXPERIMENTAL SECTION}

\section{Materials synthesis}

Graphene oxide (GO) was chemically synthesized from natural graphite flakes according to a modified Hummers method $[27,28]$.

The mesoporous polypyrrole-based graphene oxide (mPPy@GO) nanosheets were first synthesized by in-situ polymerization of pyrrole on GO surface using poly (ethylene oxide)- $b$-poly(propylene oxide)- $b$-poly(ethylene oxide) triblock copolymers (P123) as soft template. Typically, $0.2 \mathrm{~g}$ of $\mathrm{P} 123$ was dissolved in $1 \mathrm{~mL}$ tetrahydrofuran and $8 \mathrm{~mL}$ deionized water to generate aggregation. After stirring, $1.5 \mathrm{mg}$ of GO, $30 \mathrm{mg}$ of pyrrole and $12 \mathrm{mg} \mathrm{NaOH}$ were added into the solution. Then, $90 \mathrm{mg}$ of ammonium persulfate was added to initiate polymerization of pyrrole. Afterwards, mPPy@GO nanosheets were obtained after washing and centrifugation.

Through the reduction of mPPy@GO by hydrothermal treatment at $180^{\circ} \mathrm{C}$ for $12 \mathrm{~h}[29,30], m P P y @ r G O$ nanosheets were obtained. For comparison, polypyrrolebased graphene nanosheets without mesoporous structure (PPy@rGO) were also synthesized, without using P123, while other steps were kept the same as mPPy@rGO.

In order to prepare POM anchored mPPy@rGO nanosheets (mPPy@rGO-POM), 50 mg of mPPy@rGO was added to $50 \mathrm{~mL}$ of phosphomolybdic acid aqueous solution $\left(10 \mathrm{mmol} \mathrm{L}^{-1}\right)[24,31,32]$. This suspension was stirred for $2 \mathrm{~h}$ and kept for $5 \mathrm{~h}$ at room temperature. Finally, the sample was rinsed with water several times and freeze-dried to yield mPPy@rGO-POM nanosheets.

The EG nanosheets were prepared by electrochemical exfoliation of graphite in aqueous solution, as described in our previous studies [33,34].

\section{Devices assembly}

The interdigital electrodes for MSCs were directly fabricated by one-step mask-assisted technique we developed recently [33]. First, the stable ink of $2 \mathrm{~mL}$ EG dispersion in ethanol solution $\left(0.05 \mathrm{mg} \mathrm{mL}^{-1}\right)$ was vacuum filtrated as current collectors on nylon membrane $(0.22 \mu \mathrm{m}$, Agela Technologies Ltd.), with the help of a customized interdigital mask (8 interdigital fingers with $15 \mathrm{~mm}$ length, $1 \mathrm{~mm}$ width, and $0.5 \mathrm{~mm}$ width of interspacing). Then, $2 \mathrm{~mL}$ dispersion of PPy@rGO $\left(0.25 \mathrm{mg} \mathrm{mL}^{-1}\right)$, mPPy@rGO (0.25 mg mL $\left.{ }^{-1}\right)$ or mPPy@rGO-POM $\left(0.34 \mathrm{mg} \mathrm{mL}^{-1}\right)$ in aqueous solution was immediately added onto the top of the EG layer and continuously filtrated. Subsequently, the same volume of EG dispersion was filtered to form the second EG layer on the top of the active material layer. After removing the mask, the interdigital patterns of film electrodes of PG, mPG or mPGM, respectively, 


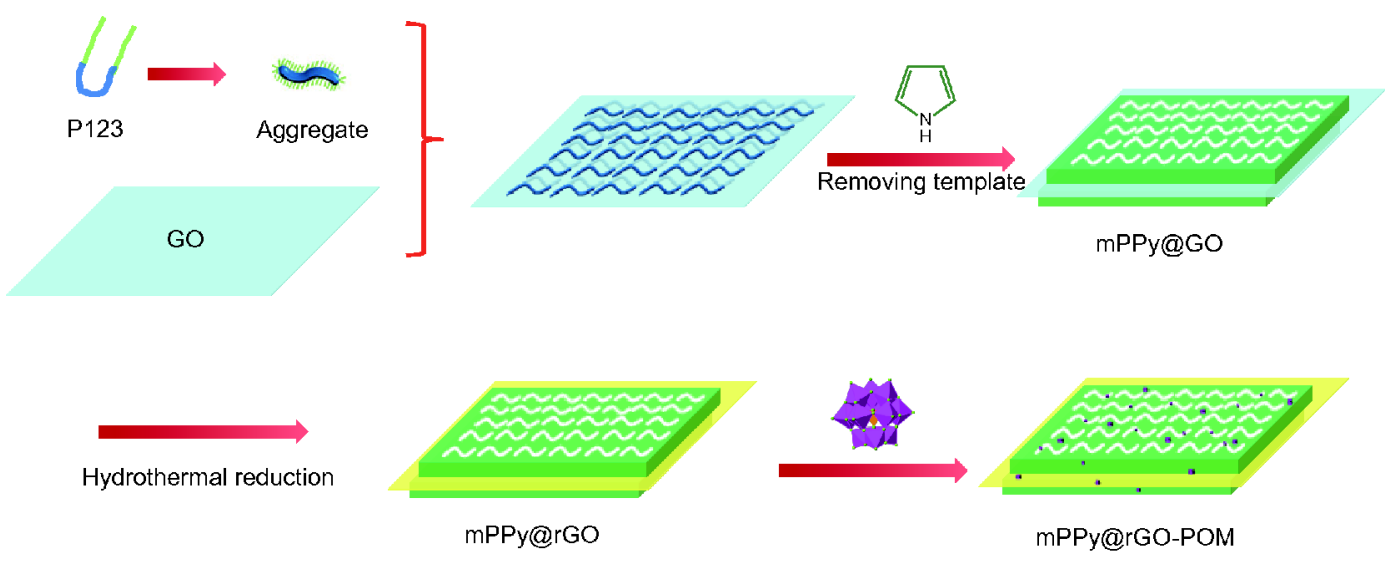

Figure 1 Scheme of the fabrication of mPPy@rGO-POM nanosheets.

were obtained. After drop-casting and solidification of gel electrolyte of polyvinyl alcohol (PVA) $/ \mathrm{H}_{2} \mathrm{SO}_{4}[35,36]$, the resulted MSCs were correspondingly denoted as PGMSCs, mPG-MSCs and mPGM-MSCs, respectively. For comparison, we also constructed the EG-MSCs based on the deposition of EG dispersion of $8 \mathrm{~mL}$, while other steps were kept the same as MPGM-MSCs.

\section{Materials characterization}

The morphology and structure of as-obtained nanosheets were characterized by scanning electron microscopy (SEM, JSM-7800F), transmission electron microscopy (TEM), high-resolution TEM (HRTEM, JEM-2100), atomic force microscopy (AFM, Veeco nanoscope multimode II-D), physical adsorption instrument (Quadrasorb SI), Raman spectrometer (LabRAM HR 800, $532 \mathrm{~nm}$ ), Fourier transform infrared spectrometer (FTIR, Bruker Optics hyperion 3000) and thermogravimetric analysis (TGA, STA 449 F3). The thickness of the film electrodes was measured using a surface profiler (Veeco Dektak 150). The electrical conductivity of film electrodes was examined by a standard four-point probes system (RTS-9).

\section{Electrochemical measurement}

The electrochemical properties of PPy@rGO,mPPy@rGO and mPPy@rGO-POM nanosheets were measured using an electrochemical workstation (CHI 760E) in a three-electrode system. The working electrode was prepared by mixing $80 \mathrm{wt} . \%$ active material, $10 \mathrm{wt} . \%$ ketjen black and 10 wt.\% poly(tetrafluoroethylene) in ethanol. The working electrode was evaluated in $1 \mathrm{~mol} \mathrm{~L}^{-1} \mathrm{H}_{2} \mathrm{SO}_{4}$ as electrolyte, using platinum plate as counter electrode and saturated calomel electrode (SCE) as reference electrode. The cyclic voltammetry $(\mathrm{CV})$ and galvanostatic charge/discharge (GCD) measurements were performed with a potential range from -0.2 to $0.8 \mathrm{~V} v$ s. SCE.

The electrochemical properties of all-solid-state PGMSCs, mPG-MSCs, mPGM-MSCs and EG-MSCs, using $\mathrm{PVA} / \mathrm{H}_{2} \mathrm{SO}_{4}$ gel electrolyte, were also measured by $\mathrm{CV}$ curves at different scan rates from 1 to $100 \mathrm{mV} \mathrm{s}^{-1}$, GCD profiles at varying current densities, and electrochemical impedance spectroscopy (EIS) in the frequency range from $0.01 \mathrm{~Hz}$ to $100 \mathrm{kHz}$ using $\mathrm{CHI} 760 \mathrm{E}$ electrochemical workstation.

\section{RESULTS AND DISCUSSION}

Fig. 1 shows the schematic of the synthesis of $2 \mathrm{D}$ mPPy@rGO-POM nanosheets via self-assembly of triblock copolymer (P123), in-situ polymerization of pyrrole monomer and anchoring of redox POM clusters. First, free-standing GO nanosheets were employed as 2D substrates for bottom-up patterning of mesoporous polypyrrole layers. In this case, the oxygen-bearing groups of GO surface primarily interacted with P123 micelles driven by hydrogen bonding to form wormlike islands. Then, pyrrole monomers were polymerized rounding building blocks of micelles. After removing the P123 template and hydrothermal reduction, mPPy@rGO nanosheets were attained. Finally, the redox POM clusters were uniformly immersed into the mesopores, and strong anchoring on the surface of mPPy@rGO nanosheets.

The morphology and structure of as-prepared mPPy@GO nanosheets were firstly characterized by SEM, TEM, AFM, and $\mathrm{N}_{2}$ adsorption-desorption isotherm measurements. As shown in Fig. 2a, b, mPPy@GO nanosheets presented uniform, ultrathin, flat and graphenelike structure with wormlike mesopores, demonstrative of successful replication of well-defined polypyrrole patterns from the vacant spacing between the GO and P123 mi- 

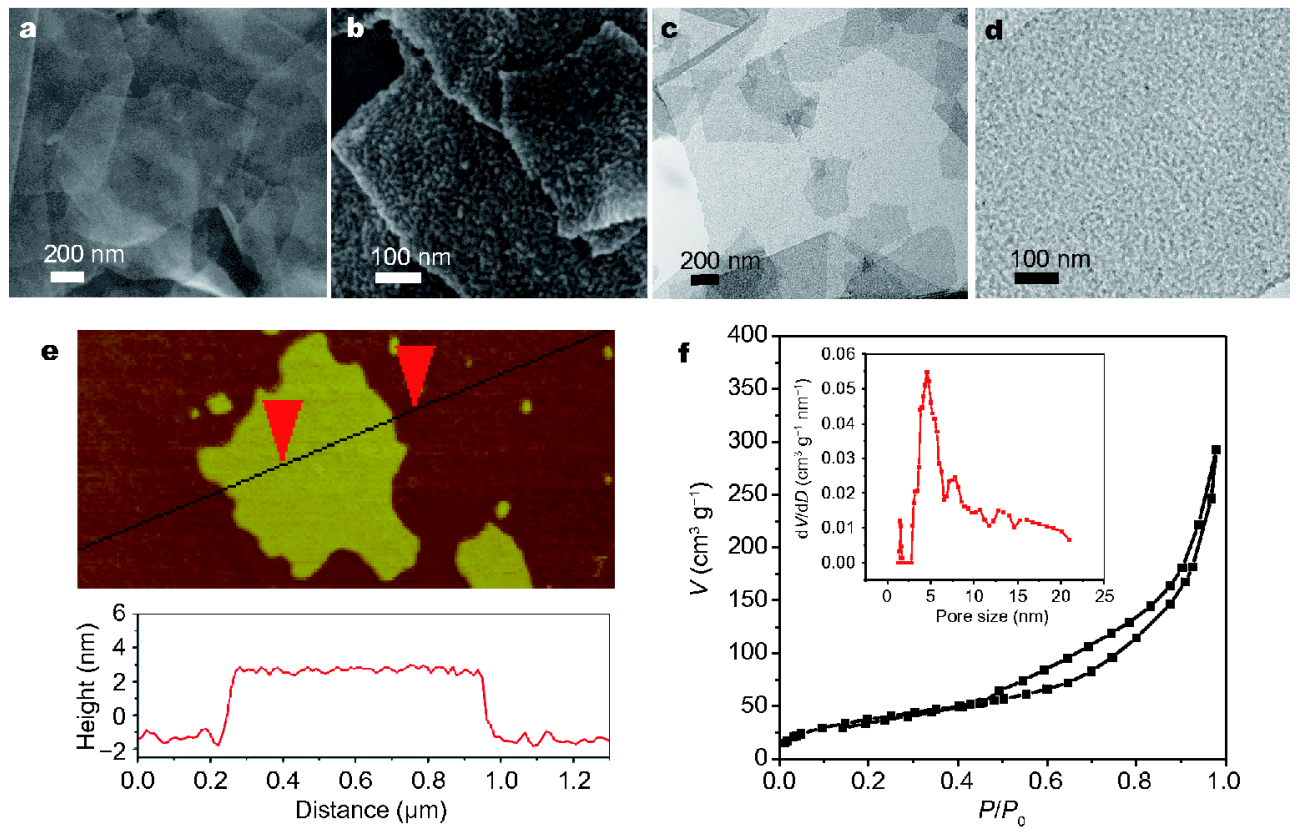

Figure 2 Morphology and structure of mPPy@GO nanosheets. (a, b) SEM images. (c, d) TEM images. (e) AFM image (up) and corresponding height profile (down). (f) $\mathrm{N}_{2}$ adsorption-desorption isotherm and pore size distribution (inset).

celles. The homogeneous mesoporous structures were also demonstrated by TEM images (Fig. $2 c$, d). The typical AFM image and height analysis (Fig. 2e) revealed ultrathin structure of mPPy@GO nanosheets with a uniform thickness of $\sim 3.7 \mathrm{~nm}$. The mesoporous feature of mPPy@GO nanosheets was further confirmed by $\mathrm{N}_{2}$ physisorption measurement (Fig. 2f). The adsorptiondesorption isotherm displayed a type IV curve with a $\mathrm{H}_{2}-$ type hysteresis loop [37]. The specific surface area and pore volume were calculated to be $142 \mathrm{~m}^{2} \mathrm{~g}^{-1}$ and $0.44 \mathrm{~cm}^{3} \mathrm{~g}^{-1}$, respectively. The narrow pore size distribution was mainly presented ranging from 5 to $10 \mathrm{~nm}$ for mPPy@GO nanosheets, consistent with SEM and TEM observations.

After reduction of mPPy@GO nanosheets by hydrothermal treatment, the resulting mPPy@rGO nanosheets, imaged by TEM characterization (Fig. 3a, b), revealed the similar ultrathin and wormlike mesoporous structure to mPPy@GO. In a sharp contrast, heterogeneous and nonporous polypyrrole was observed on the surface of graphene nanosheets (PPy@rGO, Fig. S1) without the usage of structure-directing P123, highlighting the crucial role of P123 template for the formation of mesopores. Furthermore, after anchoring POM clusters, the resultant mPPy@rGO-POM nanosheets still well retained the wormlike mesoporous structure (Fig. 3c). HRTEM image disclosed the uniform distribution of POM clusters, with ultrasmall sizes of 1-2 nm (Fig. 3d) [23], homogeneously anchored on the surfaces and in the pores of mPPy@rGO nanosheets, and the corresponding EDX analysis determined the presence of Mo, $\mathrm{P}$ and $\mathrm{O}$ elements of POM, and $\mathrm{C}, \mathrm{N}$ and $\mathrm{O}$ elements corresponding to the graphene and polypyrrole, respectively, derived from mPPy@rGOPOM nanosheets (Fig. 3e). Raman spectra of mPPy@GO, mPPy@rGO and mPPy@rGO-POM nanosheets were given in Fig. 3f. The $D$ and $G$ peaks of graphene appearing at 1350 and $1590 \mathrm{~cm}^{-1}$ were partially overlapped with the peaks of polypyrrole at 1340 and $1570 \mathrm{~cm}^{-1}$ ascribed to $\mathrm{C}=\mathrm{C}$ stretching modes $[16,22]$. The relative intensity ratio $I_{\mathrm{D}} / I_{\mathrm{G}}$ of $\mathrm{D}$ and $\mathrm{G}$ bands was 1.03 for mPPy@GO and 1.06 for mPPy@rGO, indicative of the reduction of GO $[29,35]$. Four main peaks presented at 997, 826, 667 and $287 \mathrm{~cm}^{-1}$ evidenced the existence of POM clusters (Fig. S2) in mPPy@rGO-POM, demonstrative of the integration of POM into mPPy@rGO nanosheets. In addition, FT-IR spectra of mPPy@rGO and mPPy@rGO-POM exhibited characteristic functional groups of polypyrrole vibration peaks, and Mo-O stretching modes (around $1000 \mathrm{~cm}^{-1}$ ) for mPPy@rGO-POM (Fig. S3) [38]. The weight content of POM clusters anchored on mPPy@rGO-POM nanosheets was determined to be 26.5 wt.\%, derived from the TGA curve (Fig. S4) [22,38].

Due to the unique sandwich-like structure, high-conductive graphene, mesoporous polypyrrole patterns and 

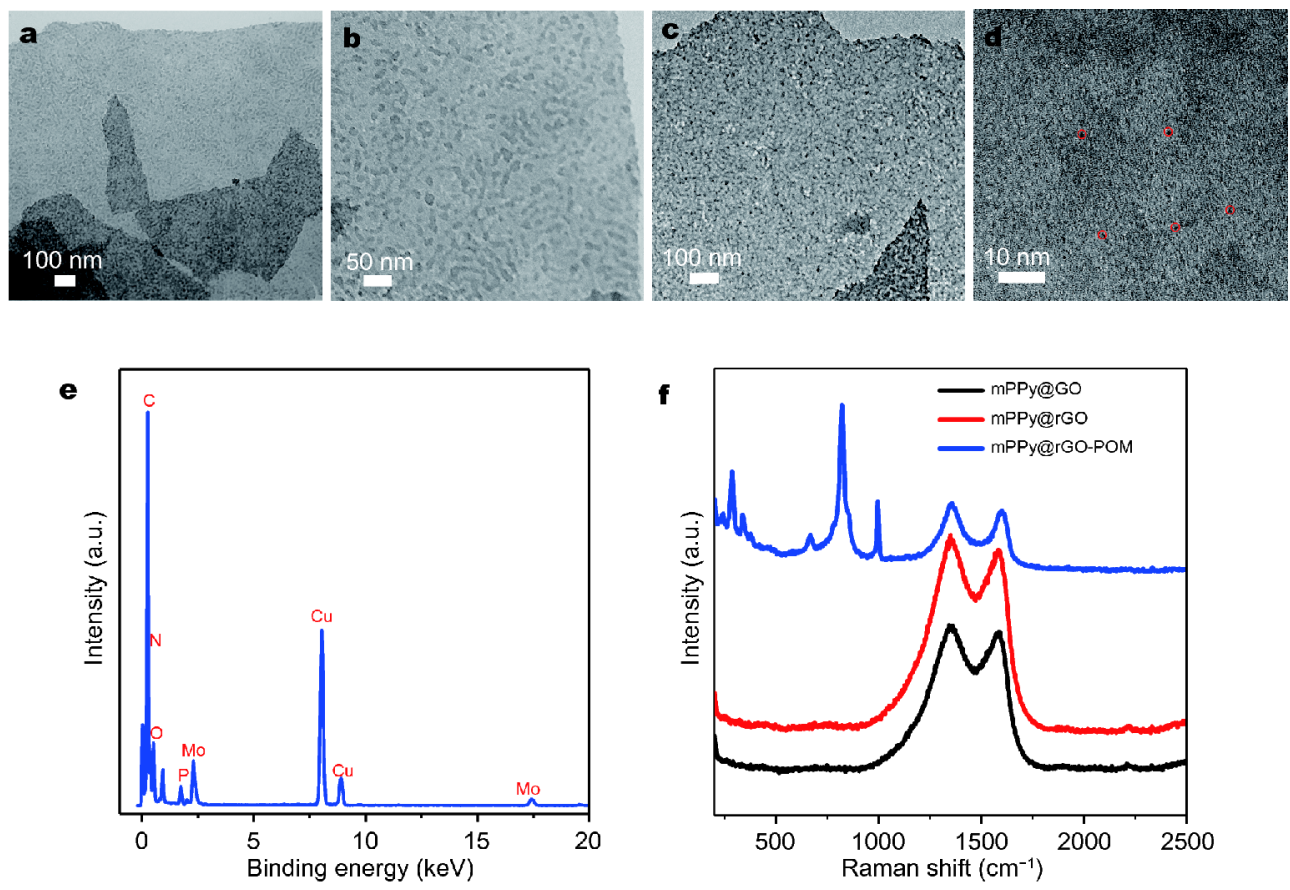

Figure 3 (a, b) TEM images of mPPy@rGO nanosheets after reduction. (c, d) TEM and HRTEM images of mPPy@rGO-POM nanosheets. (e) EDX spectrum of mPPy@rGO-POM nanosheets. Cu element is derived from TEM grid used. (f) Raman spectra of mPPy@GO,mPPy@rGO and mPPy@rGO-POM nanosheets.

uniform POM modulation, the as-obtained mPPy@rGOPOM nanosheets were expected to serve as a novel class of promising electrode materials for MSCs. To this end, we firstly evaluated their electrochemical properties of PPy@rGO, mPPy@rGO and mPPy@rGO-POM nanosheets in a three-electrode configuration (Fig. S5). Notably, both CV and GCD curves of mPPy@rGO-POM nanosheets showed strong pseudocapacitive behaviors of polypyrrole and redox POM species, in comparison with mPPy@rGO and PPy@rGO. It is worth noting that, although mPPy@rGO-POM nanosheets delivered similar gravimetric capacitance to mPPy@rGO,mPPy@rGOPOM nanosheets with high mass density of POM anchoring into mesopores hold enormous potentials for compact MSCs in term of volumetric performance metrics.

To demonstrate this assumption of constructing the compact film, we selected the pseudocapacitive mPPy@rGO-POM nanosheets as the core active materials and EG nanosheets as both film-assisted agents and metal-free current collectors, and employed an interdigital mask-assisted technique to fabricate the interdigital films (mPGM) for mPGM-MSCs. Specifically, the stable inks of EG (0.05 mg mL ${ }^{-1}$ in ethanol, $\left.2 \mathrm{~mL}\right)$, mPPy@rGO-POM $\left(0.34 \mathrm{mg} \mathrm{mL}^{-1}\right.$ in deionized water, $\left.2 \mathrm{~mL}\right)$ and $\mathrm{EG}$ $\left(0.05 \mathrm{mg} \mathrm{mL}^{-1}\right.$ in ethanol, $\left.2 \mathrm{~mL}\right)$ were continuously deposited in a controlled sequence [33]. Remarkably, the resulting interdigital mPGM film exhibited a sandwichlike structure of EG/mPPy@rGO-POM/EG, outstanding uniformity, immense continuity, robust mechanical flexibility (Fig. 4a, b), high mass density of $0.58 \mathrm{~g} \mathrm{~cm}^{-3}$ and excellent electrical conductivity of $43-162 \mathrm{~S} \mathrm{~cm}^{-1}$ (Fig. S6), and thereby can be directly employed as binder- or additive-free planar electrodes, without metal-based current collectors, for mPGM-MSCs. It is noted that the EG nanosheets are flat, transparent and ultrathin, with a layer number of $\leq 3$ (Fig. 4c, d). The resultant sandwich-like mPGM film possessed well-developed ionic and electronic networks, in which the rich mesoporous arrays could enhance the ionic diffusion, and two EG layers serve as the main conductive backbones and the rGO nanosheets were as branch-like conductive pathways for speeding electron transport (Fig. 4e). Top-view SEM images of mPGM film revealed highly uniformity, large-area continuity, and excellent structural integrity of the EG layer (Fig. 4f). Cross-section SEM images clearly exhibited micrometer thickness, and compactly layer-stacked sandwich-like structure of EG/mPPy@rGO-POM/EG (Fig. 4g, h). The homogeneous distribution and dense arrangement of mPPy@rGO-POM nanosheets were fur- 

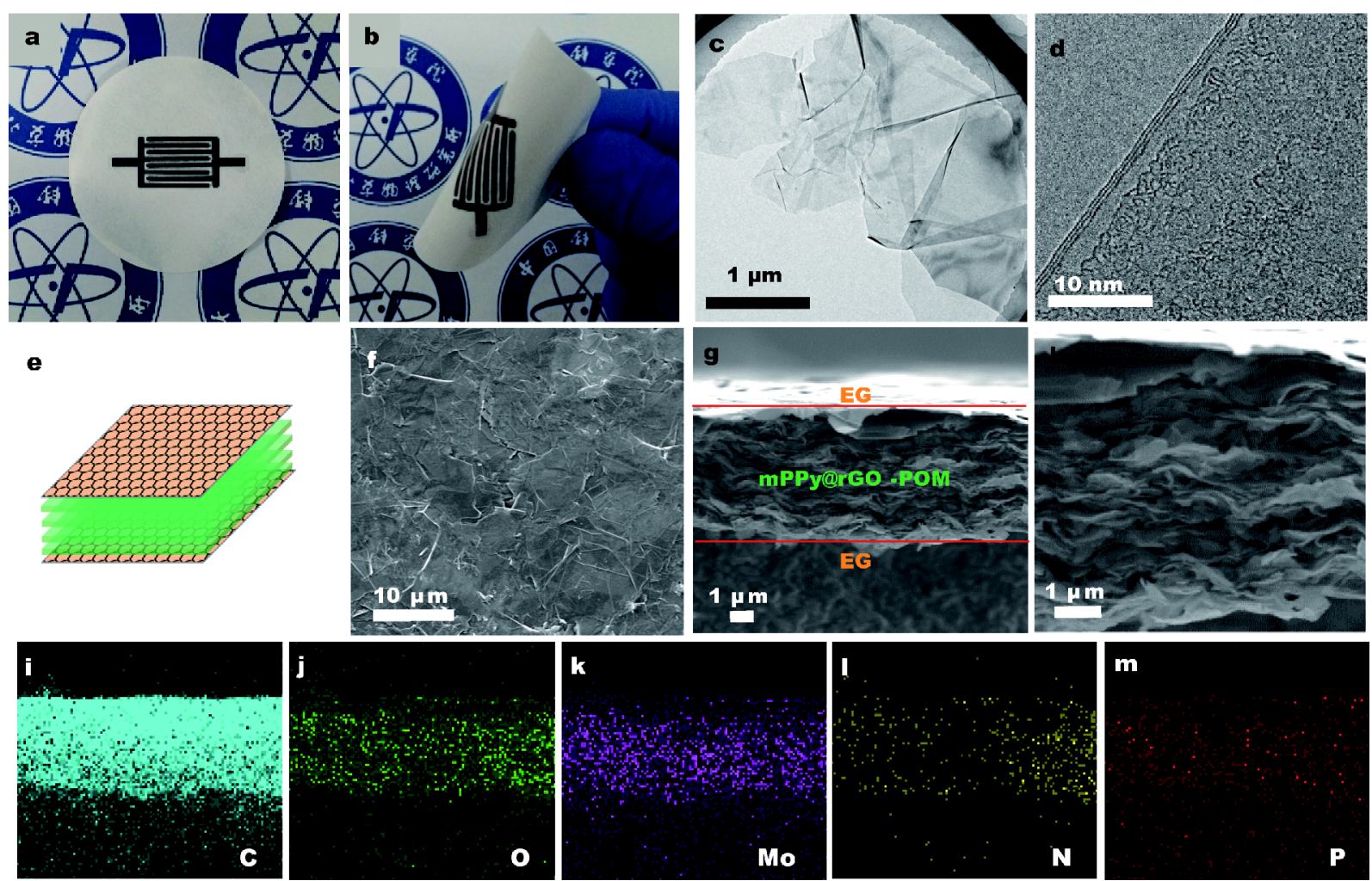

Figure $4(a, b)$ Photographs of mPGM film taken at (a) flat and (b) bending states. (c, d) TEM and HRTEM images of EG nanosheets. (e) Crosssection schematic of sandwich-like mPGM film, showing an EG/mPPy@rGO-POM/EG structure. (f) Top-view SEM image of mPGM film. (g, h) Cross-section SEM images of mPGM film at low and high magnification. (i-m) Cross-section elemental mapping analyses of mPGM film, showing the presence of (i) C, (j) O, (k) Mo, (l) N and (m) P elements.

ther validated by elemental mapping analysis from crosssection of sandwich-like mPGM film (Fig. 4i-m). After drop-casting and solidification of $\mathrm{PVA} / \mathrm{H}_{2} \mathrm{SO}_{4}$ gel electrolyte onto the interdigital electrodes, all-solid-state mPGM-MSCs were obtained.

To highlight the superiority of mesoporous structure and POM anchoring of mPPy@rGO-POM nanosheets on the improvement of electrochemical performance, we also adopted the same procedure to construct the PG-MSCs based on the sandwich-like film of EG/PPy@rGO/EG, and mPG-MSCs based on EG/mPPy@rGO/EG film, while other steps were kept the same as MPGM-MSCs. Fig. 5a shows the CV curves of all-solid-state mPGMMSCs, mPG-MSCs, and PG-MSCs tested at $10 \mathrm{mV} \mathrm{s}^{-1}$. It can be observed that the CV curves of mPGM-MSCs, in comparison with mPG-MSCs and PG-MSCs, exhibited the impressive pseudocapacitive behavior originating from redox POM clusters, and the increased current integration (Fig. 5a). The areal capacitance and volumetric capacitance of mPGM-MSCs, mPG-MSCs and PG-MSCs as a function of scan rate were presented in Fig. 5b, c.
Apparently, mPGM-MSCs delivered high areal capacitance of $115 \mathrm{mF} \mathrm{cm}^{-2}$ and outstanding volumetric capacitance of $137 \mathrm{~F} \mathrm{~cm}^{-3}$ at $1 \mathrm{mV} \mathrm{s}^{-1}$, both of which were much higher than those values of mPG-MSCs (79 $\mathrm{mF} \mathrm{cm}{ }^{-2}$ and $\left.95 \mathrm{~F} \mathrm{~cm}^{-3}\right)$, PG-MSCs $\left(74 \mathrm{mF} \mathrm{cm}^{-2}\right.$ and 68 $\left.\mathrm{F} \mathrm{cm}^{-3}\right)$, and EG-MSCs $\left(0.4 \mathrm{mF} \mathrm{cm}^{-2}\right.$ and $2.2 \mathrm{~F} \mathrm{~cm}^{-3}$, Fig. S7). Meanwhile, the mPGM-MSCs, even tested at scan rate of $100 \mathrm{mV} \mathrm{s}^{-1}$, still presented higher volumetric capacitance of $52 \mathrm{~F} \mathrm{~cm}^{-3}$ than those of mPG-MSCs $\left(44 \mathrm{~F} \mathrm{~cm}^{-3}\right)$ and PG-MSCs $\left(30 \mathrm{~F} \mathrm{~cm}^{-3}\right)$. As a result, mPGM-MSCs offered higher energy density (4.8 $\left.\mathrm{mW} \mathrm{h} \mathrm{cm}{ }^{-3}\right)$ and power density $\left(645.1 \mathrm{~mW} \mathrm{~cm}^{-3}\right)$ than those of mPG-MSCs $\left(3.3 \mathrm{~mW} \mathrm{~h} \mathrm{~cm}{ }^{-3}\right.$ and 544.2 $\mathrm{mW} \mathrm{cm} \mathrm{cm}^{-3}$ ), and PG-MSCs $\left(2.4 \mathrm{~mW} \mathrm{~h} \mathrm{~cm}{ }^{-3}\right.$ and 380.4 $\mathrm{mW} \mathrm{cm} \mathrm{cm}^{-3}$ ) in Fig. 5d. These results revealed that the presence of mesoporous patterns and further anchoring of redox POM clusters could significantly enhance the electrochemical energy storage of MSCs.

To further evaluate the electrochemical properties of mPGM-MSCs as a function of mPGM film thickness, we investigated the CV curves of mPGM-MSCs based on the 

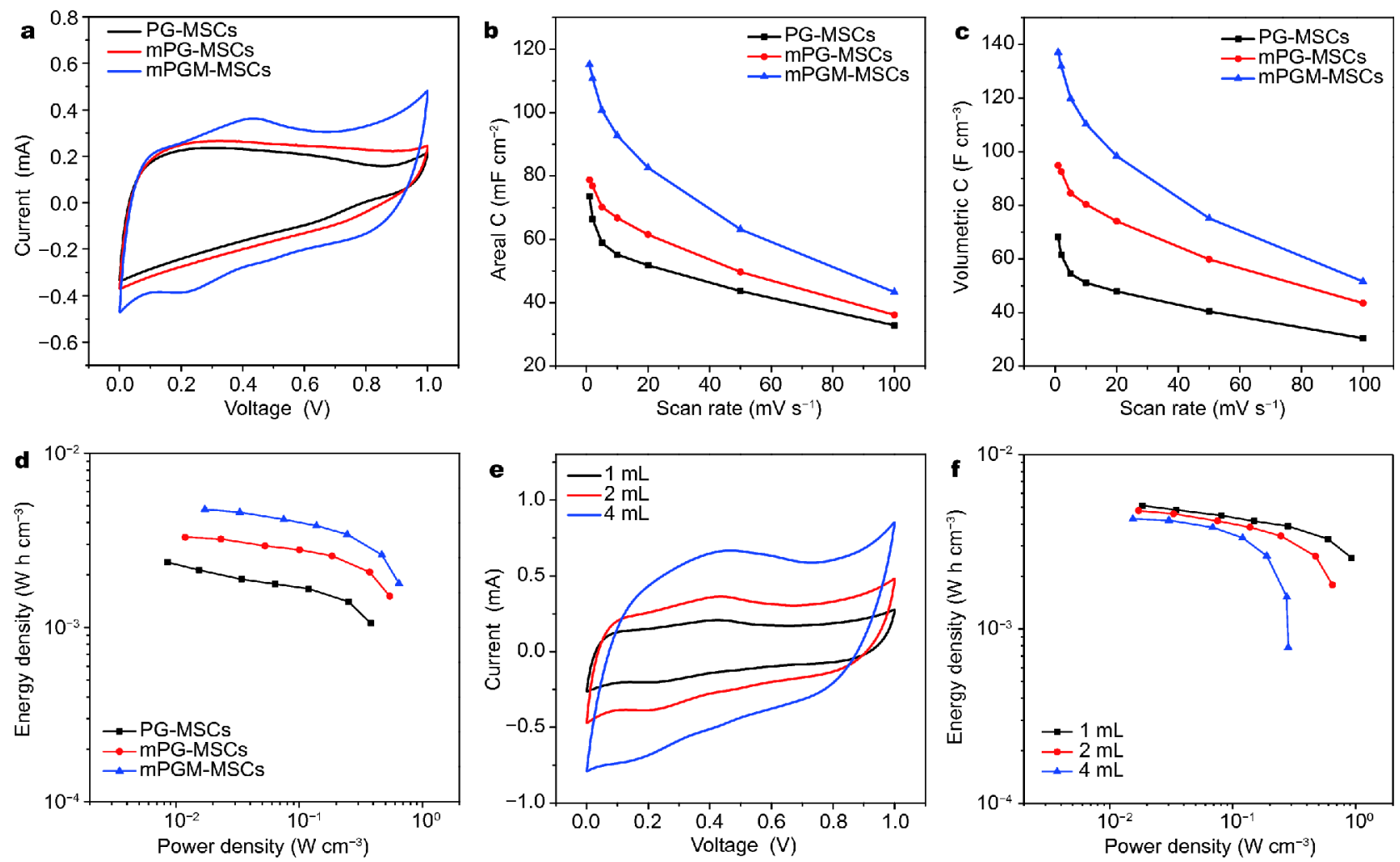

Figure 5 (a) CV curves tested at $10 \mathrm{mV} \mathrm{s}^{-1}$, (b) areal capacitance, (c) volumetric capacitance as a function of scan rate, and (d) Ragone plot of mPGMMSCs, mPG-MSCs, and PG-MSCs. (e) CV curves and (f) Ragone plot of mPGM-MSCs based on the varying volume from 1, 2 to 4 mL mPPy@rGOPOM ink.

varying volume of 1,2, and $4 \mathrm{~mL}$ mPPy@rGO-POM ink (Fig. 5e). Correspondingly, the resultant thickness of mPGM film is $4.3,8.4$ and $16.7 \mu \mathrm{m}$, respectively. It is disclosed that these three mPGM-MSCs showed the stepwise increased areal capacitance at low scan rate (e.g., 1-20 $\mathrm{mV} \mathrm{s}^{-1}$ ) while the volumetric capacitance decreased shortly, in particular, at high scan rate (e.g., $100 \mathrm{mV} \mathrm{s}^{-1}$ ) (Fig. S8). When $1 \mathrm{~mL}$ ink of mPPy@rGO-POM was used, mPGM-MSCs presented lower areal capacitance of $63 \mathrm{mF}$ $\mathrm{cm}^{-2}$ and high volumetric capacitance of $147 \mathrm{~F} \mathrm{~cm}^{-3}$ at scan rate of $1 \mathrm{mV} \mathrm{s}^{-1}$, and better rate capability than those of mPGM-MSCs based on $2 \mathrm{~mL}$ ink $\left(115 \mathrm{mF} \mathrm{cm}^{-2}\right.$ and $137 \mathrm{~F} \mathrm{~cm}^{-3}$ at $\left.1 \mathrm{mV} \mathrm{s}^{-1}\right)$ and $4 \mathrm{~mL}$ ink $\left(206 \mathrm{mF} \mathrm{cm}^{-2}\right.$ and $123 \mathrm{~F} \mathrm{~cm}^{-3}$ at $1 \mathrm{mV} \mathrm{s}^{-1}$ ). This phenomenon could be well explained by high electrical conductivity of thinner mPGM film (Fig. S6), resulting in higher electron transport and volumetric energy density of compact mPGMMSCs (Fig. 5f).

To validate the outstanding mechanical flexibility, we further evaluated the electrochemical performance of mPGM-MSCs under different bending degrees [39]. Due to high flexibility of 2D nanosheet-based electrodes, our
mPGM-MSCs exhibited impressive repeatability under different bending degrees and retained $\sim 96 \%$ of initial capacitance when the cell was tested at highly bending angle of $180^{\circ}$ (Fig. 6a, b). Moreover, mPGM-MSCs disclosed good cycling stability, showing $80 \%$ of capacitance retention after 2000 cycles (Fig. S9). To satisfy the growing demands for smart integrated electronics, we further fabricated the integrated mPGM-MSCs packing from three parallelly or serially interconnected devices to boost the capacitance or voltage output (Fig. 6c-f) [40]. As shown in Fig. $6 \mathrm{c}$, d, both $\mathrm{CV}$ and GCD curves of parallelly-connected mPGM-MSCs from device 1 to 3 presented a stepwise linear increase in the capacitance, while the integrated mPGM-MSCs connected in series illustrated the progressive voltage extension from 1.0 to $3.0 \mathrm{~V}$ (Fig. 6e,f). Dramatically, our three serially-connected mPGM-MSCs could readily power a red light emitting diode, highlighting the enormous potentiality for smart power source units with variable current and voltage outputs.

Therefore, the exceptional performance of all-solidstate planar mPGM-MSCs can be attributed to the ela- 

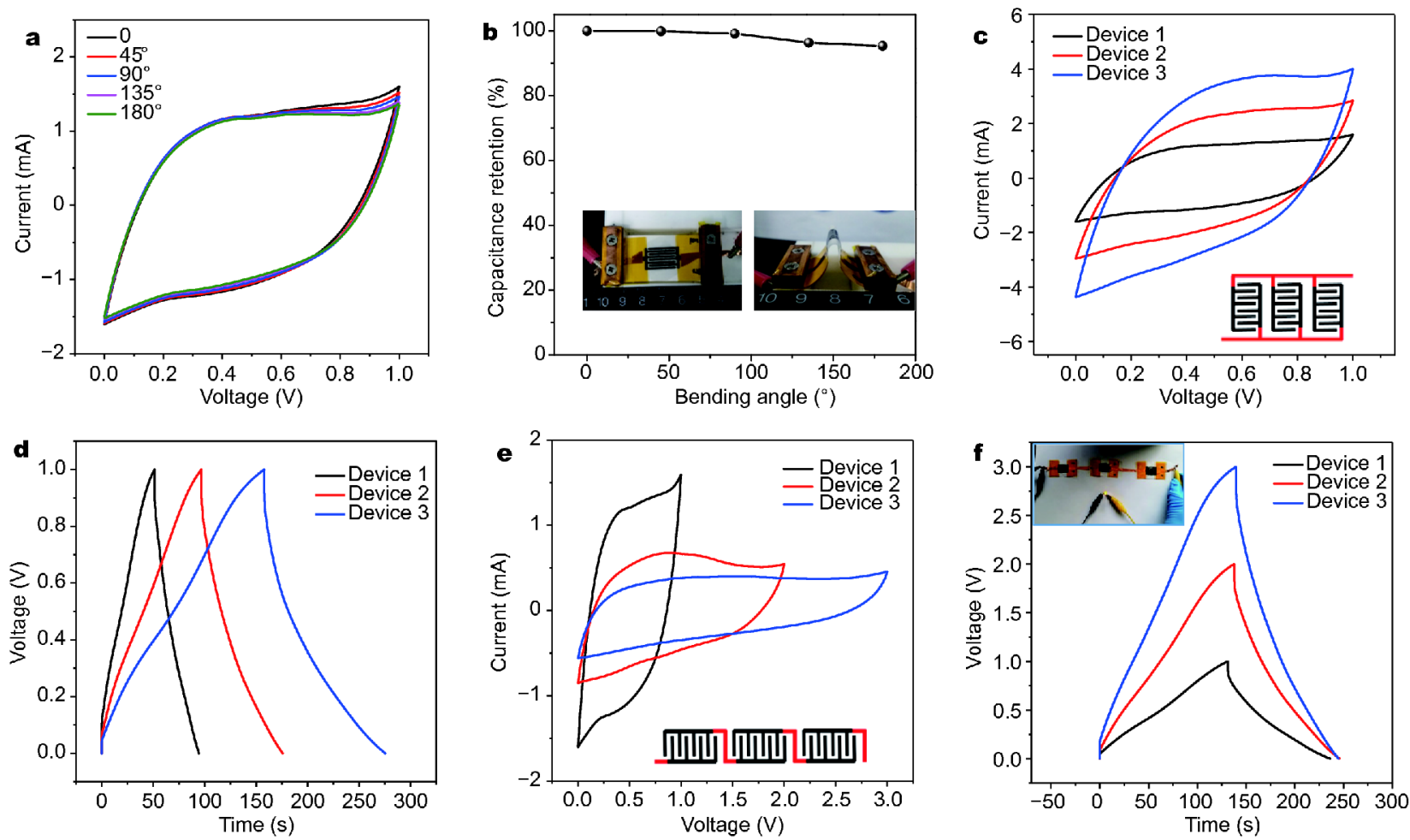

Figure 6 (a) CV curves measured at $50 \mathrm{mV} \mathrm{s}^{-1}$ of mPGM-MSCs under different bending angles, and (b) capacitance retention as a function of bending angle of mPGM-MSCs. Insets in (b) are optical images of mPGM-MSCs taken at 0 and $180^{\circ}$. (c) CV curves tested at $50 \mathrm{mV} \mathrm{s}^{-1}$ and (d) GCD profiles obtained at $0.5 \mathrm{~mA} \mathrm{~cm}^{-2}$ of three parallelly-connected mPGM-MSCs. (e) CV curves at $50 \mathrm{mV} \mathrm{s}^{-1}$ and (f) GCD profiles at $0.2 \mathrm{~mA} \mathrm{~cm}{ }^{-2}$ of three serially-connected mPGM-MSCs. Inset in (f) is a photograph of three serial mPGM-MSCs to power a light-emitting diode.

borated design of mPPy@rGO-POM nanosheets with developed mesopores and abundant redox POM clusters, and advanced fabrication of sandwich-like stacked-layer film with synergetic effect of pseudocapacitive mPPy@rGO-POM nanosheets and capacitive EG nanosheets [41]. First, mPPy@rGO-POM nanosheets feature high conductive pathways, enriched mesopores, ultrathin thickness, large surface-area-to-volume ratio for fast ion diffusion and electron transport, and uniformly anchored POM clusters for introducing large additional pseudocapacitance. Second, the densely packed film with sandwich-like structure offers higher electronic conducting networks composed of 2D capacitive EG layers and massdensity-enhanced mPPy@rGO-POM pseudocapacitive layer, more accessible active surfaces and extra interfaces to facilitate electrolyte ions transport and enhance volumetric charge storage. Last but not least, ultra-thinness and excellent flexibility of 2D nanosheets are particularly favorable for significantly improving the electrochemical performance of all-solid-state mPGM-MSCs in a planar geometry [42].

\section{CONCLUSIONS}

In summary, we have successfully synthesized a novel class of mPPy@rGO-POM nanosheets with enriched mesopore arrays and anchored redox species of POM clusters for all-solid-state planar MSCs with improved volumetric capacitance and energy density. The as-prepared 2D mPPy@rGO-POM nanosheets possessed ultrathin thickness of $\sim 3.7 \mathrm{~nm}$, wormlike mesoporous structure with a size of 5-10 nm, and uniformly anchored POM species. Furthermore, the fabricated mPGM-MSCs based on interdigital patterned films of stacked-layer mPPy@rGO-POM and EG nanosheets demonstrated impressive areal capacitance of $115 \mathrm{mF} \mathrm{cm}^{-2}$, enhanced volumetric capacitance of $137 \mathrm{~F} \mathrm{~cm}^{-3}$, excellent mechanical flexibility, and outstanding parallel or serial integration. As a consequence, our proposed strategy of synthesizing 2D mesoporous nanosheets anchored with highly redox species will open up numerous possibilities for constructing next-generation, flexible, and high-performance energy storage devices in both supercapacitors and batteries. 
Received 3 September 2017; accepted 26 September 2017; published online 26 October 2017

1 Li W, Liu J, Zhao D. Mesoporous materials for energy conversion and storage devices. Nat Rev Mater, 2016, 1: 16023

2 Dong $\mathrm{Y}, \mathrm{Wu} \mathrm{ZS}$, Ren W, et al. Graphene: a promising 2D material for electrochemical energy storage. Sci Bull, 2017, 62: 724-740

3 Wang K, Zhang X, Sun X, et al. Conducting polymer hydrogel materials for high-performance flexible solid-state supercapacitors. Sci China Mater, 2016, 59: 412-420

4 Zhang X, Cheng X, Zhang Q. Nanostructured energy materials for electrochemical energy conversion and storage: a review. J Energ Chem, 2016, 25: 967-984

5 Zhu Z, Cheng F, Hu Z, et al. Highly stable and ultrafast electrode reaction of graphite for sodium ion batteries. J Power Sources, 2015, 293: 626-634

6 Zhang C, Lv W, Tao Y, et al. Towards superior volumetric performance: design and preparation of novel carbon materials for energy storage. Energ Environ Sci, 2015, 8: 1390-1403

7 Zheng S, Wu ZS, Wang S, et al. Graphene-based materials for high-voltage and high-energy asymmetric supercapacitors. Energ Storage Mater, 2017, 6: 70-97

8 Wu ZS, Feng X, Cheng HM. Recent advances in graphene-based planar micro-supercapacitors for on-chip energy storage. Natl Sci Rev, 2014, 1: 277-292

9 Liu L, Niu Z, Chen J. Unconventional supercapacitors from nanocarbon-based electrode materials to device configurations. Chem Soc Rev, 2016, 45: 4340-4363

10 Wu ZS, Parvez K, Feng X, et al. Photolithographic fabrication of high-performance all-solid-state graphene-based planar micro-supercapacitors with different interdigital fingers. J Mater Chem A, 2014, 2: 8288-8293

11 Wang Q, Yan J, Fan Z. Carbon materials for high volumetric performance supercapacitors: design, progress, challenges and opportunities. Energ Environ Sci, 2016, 9: 729-762

12 Lin T, Chen IW, Liu F, et al. Nitrogen-doped mesoporous carbon of extraordinary capacitance for electrochemical energy storage. Science, 2015, 350: 1508-1513

13 Yang X, Cheng C, Wang Y, et al. Liquid-mediated dense integration of graphene materials for compact capacitive energy storage. Science, 2013, 341: 534-537

14 Su DS, Centi G. A perspective on carbon materials for future energy application. J Energ Chem, 2013, 22: 151-173

15 Shi Y, Peng L, Ding Y, et al. Nanostructured conductive polymers for advanced energy storage. Chem Soc Rev, 2015, 44: 6684-6696

16 Liu S, Wang F, Dong R, et al. Dual-template synthesis of 2D mesoporous polypyrrole nanosheets with controlled pore size. Adv Mater, 2016, 28: 8365-8370

17 Liu S, Wang F, Dong R, et al. Soft-template construction of 3D macroporous polypyrrole scaffolds. Small, 2017, 13: 1604099

18 Liu S, Gordiichuk P, Wu ZS, et al. Patterning two-dimensional free-standing surfaces with mesoporous conducting polymers. Nat Commun, 2015, 6: 8817

19 Wu ZS, Parvez K, Li S, et al. Alternating stacked graphene-conducting polymer compact films with ultrahigh areal and volumetric capacitances for high-energy micro-supercapacitors. Adv Mater, 2015, 27: 4054-4061

20 Yang S, Feng X, Wang L, et al. Graphene-based nanosheets with a sandwich structure. Angew Chem Int Ed, 2010, 49: 4795-4799

21 Ji Y, Huang L, Hu J, et al. Polyoxometalate-functionalized nano- carbon materials for energy conversion, energy storage and sensor systems. Energ Environ Sci, 2015, 8: 776-789

22 Suárez-Guevara J, Ruiz V, Gómez-Romero P. Stable graphenepolyoxometalate nanomaterials for application in hybrid supercapacitors. Phys Chem Chem Phys, 2014, 16: 20411-20414

23 Genovese M, Lian K. Polyoxometalate modified pine cone biochar carbon for supercapacitor electrodes. J Mater Chem A, 2017, 5: 3939-3947

24 Dubal DP, Suarez-Guevara J, Tonti D, et al. A high voltage solid state symmetric supercapacitor based on graphene-polyoxometalate hybrid electrodes with a hydroquinone doped hybrid gel-electrolyte. J Mater Chem A, 2015, 3: 23483-23492

25 Chen Y, Han M, Tang Y, et al. Polypyrrole-polyoxometalate/reduced graphene oxide ternary nanohybrids for flexible, all-solidstate supercapacitors. Chem Commun, 2015, 51: 12377-12380

26 Huang A, Yan J, Zhang H, et al. Effect of the pore length and orientation upon the electrochemical capacitive performance of ordered mesoporous carbons. J Energ Chem, 2017, 26: 121-128

27 Zhu Y, Murali S, Cai W, et al. Graphene and graphene oxide: synthesis, properties, and applications. Adv Mater, 2010, 22: 39063924

28 Wu ZS, Zhou GM, Yin LC, et al. Graphene/metal oxide composite electrode materials for energy storage. Nano Energy, 2012, 1: 107131

29 Pei S, Cheng HM. The reduction of graphene oxide. Carbon, 2012, 50: 3210-3228

30 Wu ZS, Sun Y, Tan YZ, et al. Three-dimensional graphene-based macro- and mesoporous frameworks for high-performance electrochemical capacitive energy storage. J Am Chem Soc, 2012, 134: 19532-19535

31 Hu C, Zhao E, Nitta N, et al. Aqueous solutions of acidic ionic liquids for enhanced stability of polyoxometalate-carbon supercapacitor electrodes. J Power Sources, 2016, 326: 569-574

32 Ruiz V, Suárez-Guevara J, Gomez-Romero P. Hybrid electrodes based on polyoxometalate-carbon materials for electrochemical supercapacitors. Electrochem Commun, 2012, 24: 35-38

33 Xiao $\mathrm{H}, \mathrm{Wu} \mathrm{ZS}$, Chen $\mathrm{L}$, et al. One-step device fabrication of phosphorene and graphene interdigital micro-supercapacitors with high energy density. ACS Nano, 2017, 11: 7284-7292

34 Qin J, Wu ZS, Zhou F, et al. Simplified fabrication of high areal capacitance all-solid-state micro-supercapacitors based on graphene and $\mathrm{MnO}_{2}$ nanosheets. Chin Chem Lett, 2017

35 Wang $\mathrm{S}, \mathrm{Wu} \mathrm{ZS}$, Zheng $\mathrm{S}$, et al. Scalable fabrication of photochemically reduced graphene-based monolithic micro-supercapacitors with superior energy and power densities. ACS Nano, 2017, 11: 4283-4291

36 Zheng S, Li Z, Wu ZS, et al. High packing density unidirectional arrays of vertically aligned graphene with enhanced areal capacitance for high-power micro-supercapacitors. ACS Nano, 2017, 11: 4009-4016

37 An X, Yang H, Wang Y, et al. Hydrothermal synthesis of coherent porous $\mathrm{V}_{2} \mathrm{O}_{3}$ /carbon nanocomposites for high-performance lithium- and sodium-ion batteries. Sci China Mater, 2017, 60: 717727

38 Cui Z, Guo CX, Yuan W, et al. In situ synthesized heteropoly acid/ polyaniline/graphene nanocomposites to simultaneously boost both double layer- and pseudo-capacitance for supercapacitors. Phys Chem Chem Phys, 2012, 14: 12823-12828

39 Chen C, Cao J, Lu Q, et al. Foldable all-solid-state supercapacitors integrated with photodetectors. Adv Funct Mater, 2017, 27: 
1604639

40 Liu L, Niu Z, Chen J. Design and integration of flexible planar micro-supercapacitors. Nano Res, 2017, 10: 1524-1544

$41 \mathrm{Wu}$ ZS, Zheng Y, Zheng S, et al. Stacked-layer heterostructure films of $2 \mathrm{D}$ thiophene nanosheets and graphene for high-rate allsolid-state pseudocapacitors with enhanced volumetric capacitance. Adv Mater, 2017, 29: 1602960

$42 \mathrm{Wu}$ ZS, Yang S, Zhang L, et al. Binder-free activated graphene compact films for all-solid-state micro-supercapacitors with high areal and volumetric capacitances. Energ Storage Mater, 2015, 1: $119-126$

Acknowledgements This work was supported by the National Natural Science Foundation of China (51572259), National Key R\&D Program of China (2016YBF0100100 and 2016YFA0200200), Natural Science Foundation of Liaoning Province (201602737), Recruitment Program of
Global Expert (1000 Talent Plan), DICP, China Postdoctoral Science Foundation (2016M601348), and Exploratory Research Program of Shaanxi Yanchang Petroleum (Group) Co., LTD \& DICP.

Author contributions Wu ZS proposed and supervised the whole project. Qin J and Ren R conducted the experiments and prepared the MSCs. Zhou F and Xiao H participated in synthesis of EG materials and fabrication of MSCs mask. Qin J and Wu ZS wrote and revised the manuscript. All authors contributed to the discussion and comments of this manuscript.

Conflict of interest The authors declare that they have no conflict of interest.

Supplementary information Additional detailed calculations, and figures are available in the online version of the paper.

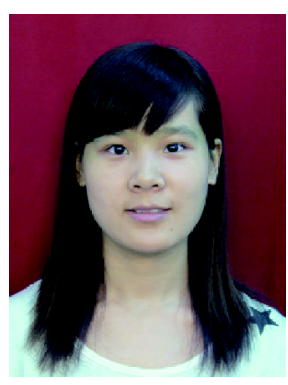

Jieqiong Qin is pursuing PhD degree in Dalian Institute of Chemical Physics (DICP), Chinese Academy of Sciences (CAS), under the supervision of Prof. Zhong-Shuai Wu. She received his MS degree in China Research Institute of Daily Chemical Industry in 2016. Her current research focuses on graphene and 2D materials for energy storage devices, such as micro-supercapacitors.

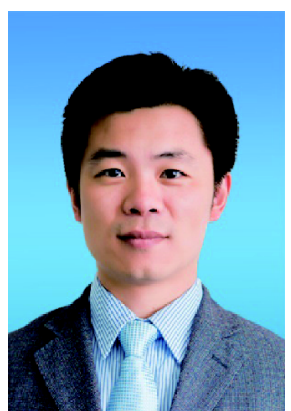

Zhong-Shuai Wu received his Ph.D in materials science from Institute of Metal Research, CAS, in 2011, and worked as a postdoctor at Max-Planck Institute for Polymer Research in 2011-2015. Then he joined DICP, CAS, and was appointed as full Professor, and group leader of 2D Materials \& Energy Devices. He has published more than 60 research articles in Adv. Mater., Nat.Commun., JACS, ACS Nano etc., with a total citation of more than 12,000 times. His research focuses on graphene and $2 \mathrm{D}$ materials for energy storage devices, such as supercapacitors, micro-supercapacitors, high-energy batteries (Li-S, Li-, K-, Na-ion), flexible and planar energy-storage devices.

\section{可控制备磷钼酸复合介孔聚吡咯/石墨烯纳米片应用于高比容量全固态微型超级电容器}

\section{秦洁琼 ${ }^{1,2}$, 周锋 $^{1}$, 肖涵 ${ }^{1}$, 任如意 ${ }^{1}$, 吴忠帅 ${ }^{1 *}$}

摘要 微型超级电容器是一种新型的、柔性化、易集成、可穿戴便携的电化学储能器件, 但提高电极材料和器件的性能仍面临着巨大的 挑战. 本文成功首次制备出一种二维磷钼酸复合介孔聚吡咯/石墨烯纳米片, 具有超薄、比表面积大、孔道结构发达、导电性好、赝电容 高的特点. 然后, 在十指交叉形状的掩模版协助下, 采用交替沉积的方法, 一步制备出电化学剥离石墨烯和磷钼酸复合介孔聚吡咯/石墨烯 纳米片三明治层状结构、高致密、高导电的复合薄膜图案化电极. 该复合薄膜图案化电极应用于全固态平面微型电容器, 表现出显著提 高的面电容 $\left(115 \mathrm{mF} \mathrm{cm}^{-2}\right.$ )和体电容 $\left(137 \mathrm{~F} \mathrm{~cm}^{-3}\right)$, 以及优异的机械柔性和可串/并联直接集成的特征. 总之, 本文通过对石墨烯基二维材料 的合理设计和微型超级电容器的高效组装实现了器件性能的极大提高, 为发展新一代的微型储能器件提供了一定的科学依据. 